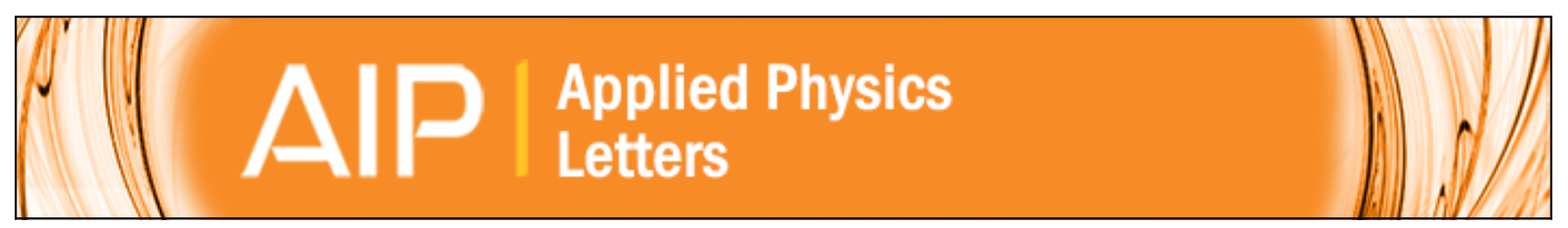

Co/Au multilayers with graded magnetic anisotropy for magnetic field sensing

M. Matczak, P. Kuwik, B. Szymaski, M. Urbaniak, M. Schmidt, J. Aleksiejew, F. Stobiecki, and A. Ehresmann

Citation: Applied Physics Letters 100, 162402 (2012); doi: 10.1063/1.4704176

View online: http://dx.doi.org/10.1063/1.4704176

View Table of Contents: http://scitation.aip.org/content/aip/journal/apl/100/16?ver=pdfcov

Published by the AIP Publishing

Advertisement:

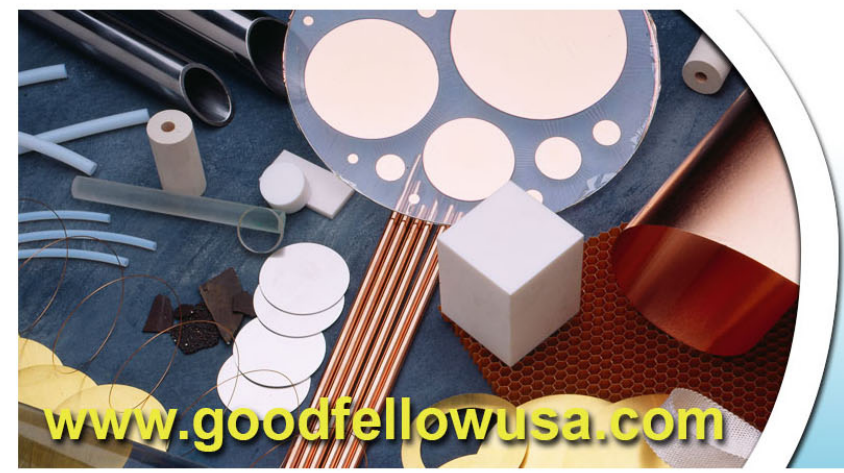

BODIFEl|OII

metals • ceramics • polymers composites • compounds • glasses

Save $5 \% \cdot$ Buy online 70,000 products $\cdot$ Fast shipping 


\title{
Co/Au multilayers with graded magnetic anisotropy for magnetic field sensing
}

\author{
M. Matczak, ${ }^{1}$ P. Kuświk, ${ }^{1}$ B. Szymański, ${ }^{1}$ M. Urbaniak, ${ }^{1}$ M. Schmidt, ${ }^{1}$ J. Aleksiejew, ${ }^{1}$ \\ F. Stobiecki, ${ }^{1}$ and A. Ehresmann ${ }^{2}$ \\ ${ }^{1}$ Institute of Molecular Physics, Polish Academy of Sciences, ul. Smoluchowskiego 17, 60-179 Poznań, Poland \\ ${ }^{2}$ Department of Physics and Center for Interdisciplinary Nanostructure Science and Technology (CINSaT), \\ University of Kassel, Heinrich-Plett-Str. 40, D-34132 Kassel, Germany
}

(Received 23 February 2012; accepted 31 March 2012; published online 17 April 2012)

\begin{abstract}
A concept of a magnetoresistive sensor for magnetic fields based on a $\mathrm{Au} / \mathrm{Co}$-wedge/Au/Co/Au multilayer is proposed. The wedged Co layer is characterized by a laterally changing coercivity resulting from a gradient of perpendicular magnetic anisotropy. Its magnetization reversal in a perpendicular magnetic field takes place by movement of a single domain wall in the direction parallel to the anisotropy gradient. The magnetization reversal of the multilayer has been investigated by magnetooptical and magnetoresistive measurements. The resistance of the proposed film system correlates well with the position of the domain wall and thus it can be used to sense magnetic fields. (C) 2012 American Institute of Physics. [http://dx.doi.org/10.1063/1.4704176]
\end{abstract}

Magnetic thin films where the magnetic anisotropy perpendicular to the sample surface varies as a function of depth below the sample surface are currently intensively investigated $^{1-6}$ due to possible applications in recording media. Films with a lateral anisotropy gradient, however, are less well studied. Nevertheless also such systems possess very interesting application possibilities, for example, for a controlled transport of magnetic particles trapped in the moving stray field of a moving domain wall or as will be shown in the present paper, as magnetic field sensors which can permanently monitor the maximum field value of a varying magnetic field. The required lateral anisotropy gradient can be created by a continuous variation of the thicknesses of the involved layers ${ }^{7}$ or by bombardment with light ions with laterally varying ion fluence. ${ }^{8}$ With both techniques, an almost constant gradient of the coercive field $\left(\mathrm{d} H_{\mathrm{C}} / \mathrm{d} x\right)$ has been achieved in, e.g., Au/Co multilayers with perpendicular magnetic anisotropy. In such a layer system, the magnetization reversal takes place by domain wall movement and the lateral position of this wall is determined by the field maximum of a homogenous external field applied perpendicularly to the sample plane. ${ }^{8}$ Here, we will show that magnetic layers with a lateral anisotropy gradient and thereby a gradient of $H_{\mathrm{C}}$ can be used as magnetic field sensors, when the proven domain wall movement in a layer with anisotropy gradient is combined with magnetoresistive read out. As a proof of principle, the sensor will be fabricated as a spin valve structure composed of two ferromagnetic layers with perpendicular anisotropy, one with laterally varying anisotropy and the other with approximately constant anisotropy, separated by a metallic non magnetic layer (see Fig. 1). As a consequence, the resistance of the system, when measured in current-in-plane configuration along the $x$ coordinate defined in Fig.1, is determined by the ratio of the lengths of the two domains in the graded layer, or in other words, by the position of the domain wall. A current induced domain wall motion has been sensed with a similar technique by Laribi. ${ }^{9}$ The motion of a single domain wall in one layer of a spin valve structure induced by a designed magnetic field pulse has been also applied in the displacement sensors. ${ }^{10,11}$ Similarly ferromagnetic layers with uniaxial perpendicular anisotropy have already been used in magnetoresistive sensors resulting in high sensitivity ${ }^{12-14}$ or linear $R(H)$ dependence. ${ }^{15,16}$ However, ferromagnetic layers with graded perpendicular anisotropy have as yet not been used in spin valve structures.

For the experiments, a (Au-2 nm/Co ${ }^{\mathrm{S}}$-wedge-0.6-1.2 nm/ $\mathrm{Au}-2 \mathrm{~nm} / \mathrm{Co}^{\mathrm{H}}-0.6 \mathrm{~nm} / \mathrm{Au}-2 \mathrm{~nm}$ ) layered system has been fabricated, which is schematically shown in Fig. 1 (superscripts $\mathrm{S}$ and $\mathrm{H}$ designate the magnetically soft and hard layers, respectively). The sample had a total length of $19 \mathrm{~mm}$ and the wedge of the $\mathrm{Co}^{\mathrm{S}}$ layer extended over $10 \mathrm{~mm}$ between $x=6 \mathrm{~mm}$ and $x=16 \mathrm{~mm}$. The thickness of the $\mathrm{Co}^{\mathrm{S}}$ layer was $0.6 \mathrm{~nm}$ for positions $x \leq 6 \mathrm{~mm}$ and $1.2 \mathrm{~nm}$ for $x \geq 16 \mathrm{~mm}$. During the same deposition process, a reference sample (Au$2 \mathrm{~nm} / \mathrm{Co}^{\mathrm{S}}$,ref wedge-0.6-1.2 nm/Au-2 nm) containing only the wedged ferromagnetic layer has been deposited. The layer systems were deposited on naturally oxidized $\mathrm{Si}(111)$ covered by a (Ti-2 nm/Au- $2 \mathrm{~nm})_{5}$ buffer using UHV magnetron sputtering. As is known from previous investigations, ${ }^{7,17-22}$

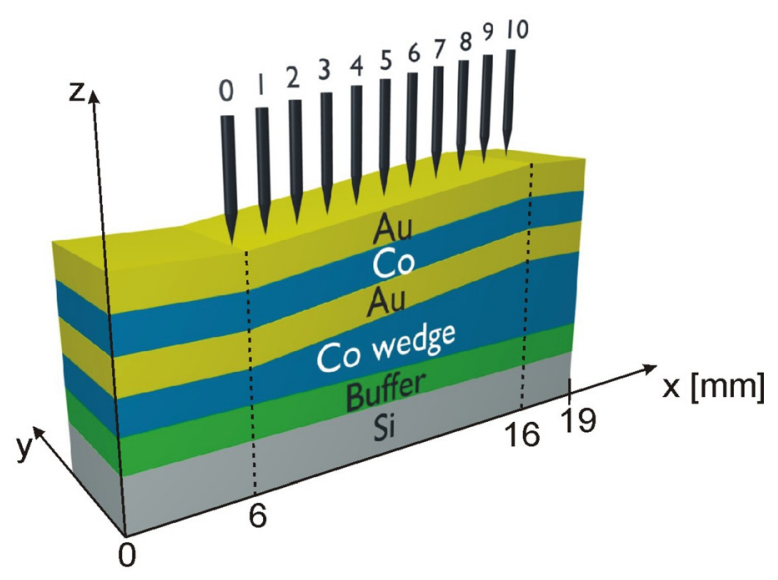

FIG. 1. Schematic representation of the spin valve structure and arrangement of contacts for magnetoresistance measurements. 
cobalt layers sandwiched between gold show perpendicular anisotropy in the thickness range between 0.3 and $1.8 \mathrm{~nm}$. For thicknesses below $0.3 \mathrm{~nm}$, Co displays superparamagnetic characteristics due to discontinuities in the layer and for thicknesses of about $1.8 \mathrm{~nm}$, a spin reorientation transition (SRT) (Ref. 21) is observed (the exact thickness where SRT occurs depends on deposition conditions and buffer). For sputter deposited polycrystalline Co layers used in the present experiments, the reorientation transition takes place at $1.5 \pm 0.3 \mathrm{~nm}$ and the maximum of $H_{\mathrm{C}}$ is observed at about $0.5 \mathrm{~nm}^{22}$ Therefore, the chosen Co wedge with $0.6 \mathrm{~nm}$ $\leq t_{\mathrm{Co}} \leq 1.2 \mathrm{~nm}$ ( $t_{\mathrm{Co}}$-thickness of the Co layer) placed between Au layers of constant thicknesses shows a monotonic decrease of $H_{\mathrm{C}}^{\mathrm{S}}$ with increasing $t_{\mathrm{Co}}$ (Fig. 2(a)). In Fig. 2(a) also the coercive fields $H_{\mathrm{C}}^{\mathrm{H}}$ and $H_{\mathrm{C}}^{\mathrm{S}}$ of the magnetically hard $\left(\mathrm{Co}^{\mathrm{H}}\right.$ layer with $\left.t_{\mathrm{Co}}=0.6 \mathrm{~nm}\right)$ and magnetically soft (wedge shaped $\mathrm{Co}^{\mathrm{S}}$ layer) layers as well as the coercive field $H_{\mathrm{C}}^{\mathrm{S} \text {,ref }}$ of the Co wedge of the reference sample as functions of the $x$ coordinate are shown. All characteristic fields have been determined from hysteresis loops measured with a polar magnetooptical Kerr effect (PMOKE) magnetometer at different positions $x$ along the spin valve structure and the reference sample. The measurements were performed at room temperature with a magnetic field sweep rate of $30 \mathrm{Oe} / \mathrm{min}$. An exemplary hysteresis loop for the spin valve system at a position $x=12 \mathrm{~mm}$ is displayed in Fig. 2(b), a loop of the reference sample at $x=12 \mathrm{~mm}$ is shown in Fig. 2(c). The

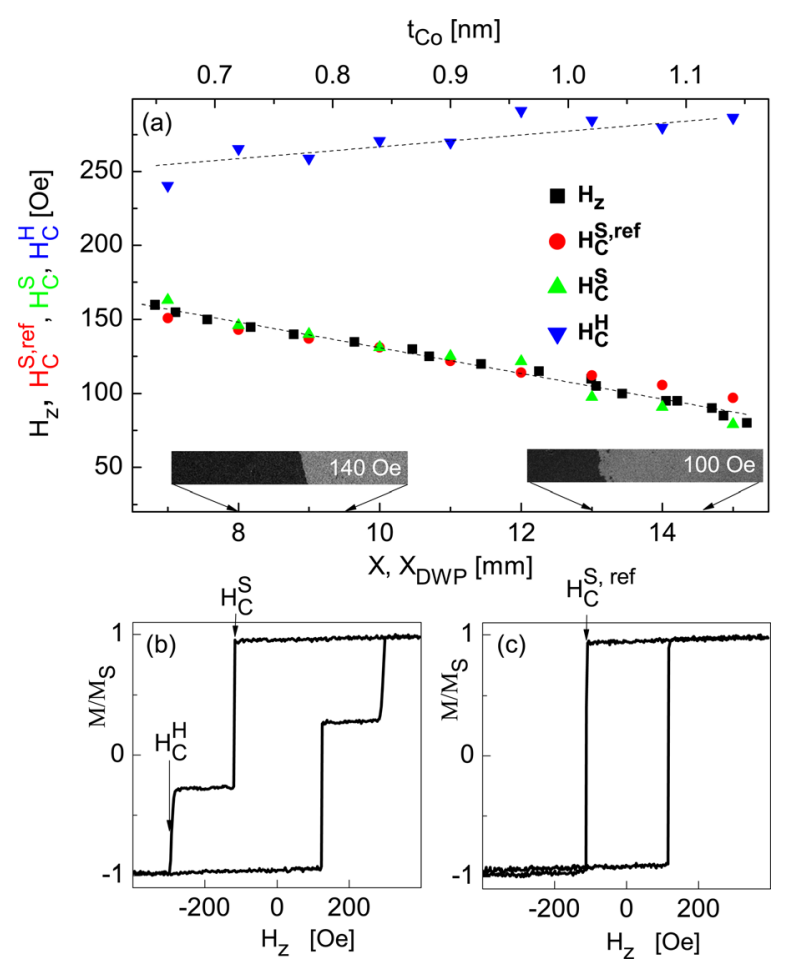

FIG. 2. (a) Coercive fields $H_{\mathrm{C}}^{\mathrm{H}}$ (down triangles) of the magnetically hard and $H_{\mathrm{C}}^{\mathrm{S}}$ (circles) of the magnetically soft layer of the complete spin valve layer system and $H_{\mathrm{C}}^{\mathrm{S}, \text { ref }}$ (up triangles) of the wedged layer of the reference sample as functions of the sample position along the wedge determined from PMOKE hysteresis loops. The domain wall positions $x_{\text {DWP }}\left(H_{\mathrm{z}}\right)$ are also shown as determined from a series of Kerr microscopy observations. Insets show Kerr microscope images around the position of the domain wall of the wedge layer after field pulses of $H_{\mathrm{z}}=140 \mathrm{Oe}$ and $H_{\mathrm{z}}=100 \mathrm{Oe}$. (b) Exemplary hysteresis loop of the spin valve layer system at $x=12 \mathrm{~mm}$. (c) Exemplary hysteresis loop of the reference layer system at $x=12 \mathrm{~mm}$. similar dependencies of $H_{\mathrm{C}}^{\mathrm{S}}(x)$ and $H_{\mathrm{C}}^{\mathrm{S}, \text { ref }}(x)$ indicate that the interaction between Co layers across the $2 \mathrm{~nm}$ Au layer is relatively weak and therefore it will, for simplicity, be neglected in the further discussion. The small increase in $H_{\mathrm{C}}^{\mathrm{H}}$ with $x$ is possibly caused by an improvement of the $\mathrm{Co}^{\mathrm{H}} / \mathrm{Au}$ layer interface with increasing thickness of the bottom (wedge shaped) $\mathrm{Co}^{\mathrm{S}}$ layer. For layer systems with a reverse order of the ferromagnetic layers (wedge shaped Co layer above the Co layer with constant thickness), $H_{\mathrm{C}}^{\mathrm{H}}$ turned out to be independent of $x$.

The magnetic domain structure of the wedged layer has been visualized by polar magnetooptical Kerr microscopy (insets of Fig. 2(a)). All images were taken at zero external magnetic field after sample saturation with a field of $H_{\mathrm{z}}=-1 \mathrm{kOe}( \pm 300 \mathrm{Oe}$ is sufficient to saturate both magnetic layers) and then applying a field pulse with $+H_{\mathrm{z}}>0$ for $30 \mathrm{~s}$ antiparallel to the saturation field. The domain wall position (DWP) $x_{\text {DWP }}$ in the wedged layer has been recorded for different fields $+H_{\mathrm{z}}$ (Fig. 2(a)). The position of the domain wall is determined by the coercivity gradient and the sample's magnetic history. Therefore, the values of $H_{\mathrm{C}}^{\mathrm{S}}(x)$ and $x_{\text {DWP }}\left(H_{\mathrm{z}}\right)$ are correlated.

The magnetoresistance has been determined using 11 point contacts (numbered from 0 to 10 in Fig. 1) along the $x$ coordinate with $1 \mathrm{~mm}$ spacing. The first and the last contacts were used to supply the current. Voltages have been measured between the other nine contacts. The resistance changes as functions of the magnetic field applied perpendicularly to the sample surface were measured by neighboring contact pairs (i.e., between pairs 1-2, 2-3,.., and 8-9) and between the outermost contacts (1-9). All magnetoresistance measurements were performed simultaneously using a multichannel voltmeter. Prior to measurement, the sample was saturated in a $-1 \mathrm{kOe}$ field. Subsequently, the field was increased with $30 \mathrm{Oe} / \mathrm{min}$ rate up to $H_{\mathrm{z}}=+300 \mathrm{Oe}$. The transitions between the low and high resistance states for the neighboring contacts are shown in Fig. 3 for 80 Oe $\leq H_{\mathrm{z}}$ $\leq 170$ Oe. The low and high resistance states correspond to parallel $\left(R_{\mathrm{P}}\right)$ and antiparallel $\left(R_{\mathrm{AP}}\right)$ magnetizations in the two ferromagnetic layers, respectively. The magnetic field interval where the transition takes place corresponds to the magnetic field interval necessary to move the domain wall over the distance between the two neighboring contacts. Due to

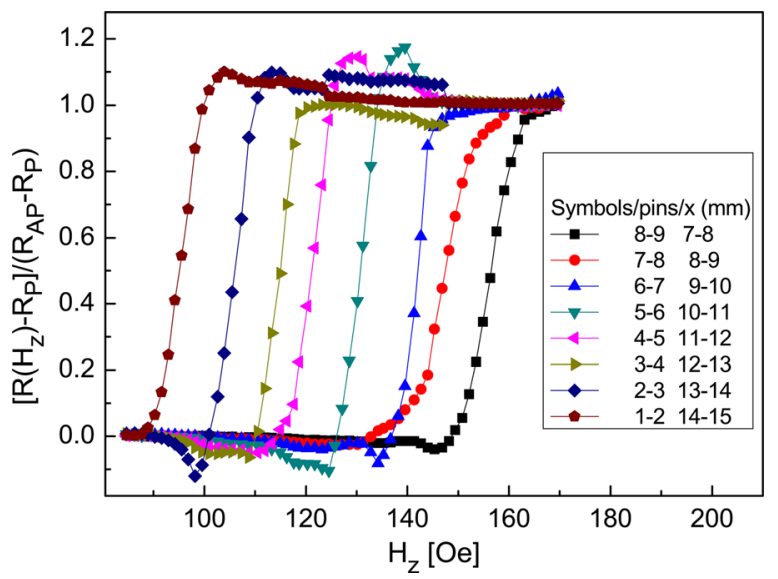

FIG. 3. Changes of resistance determined for different contact pairs (cf. Fig. 1). 

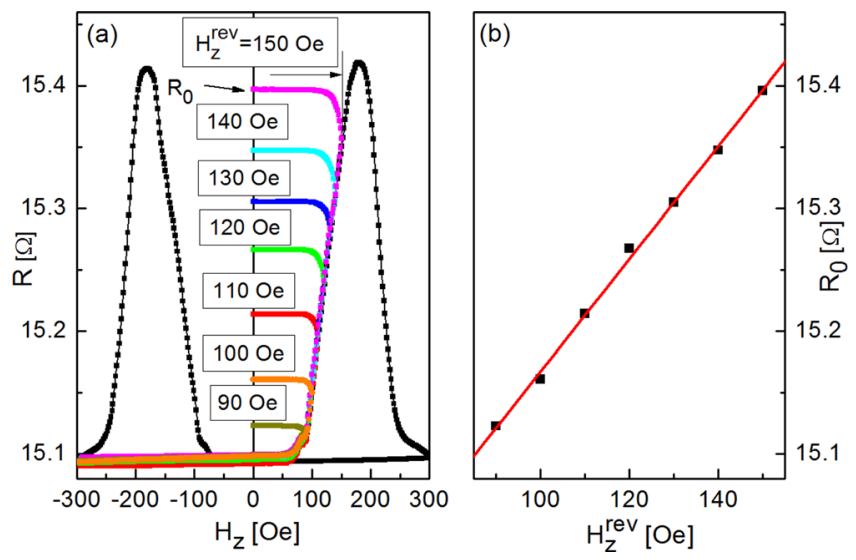

FIG. 4. (a) Major and minor magnetoresistance loops of the spin valve system. Minor loops have been taken with different values of $H_{\mathrm{z}}$ as described in the text. (b) Remanent resistance of the sample as a function of $H_{\mathrm{z}}$.

the small distance $(1 \mathrm{~mm})$ between neighboring contacts, the resistance increase occurs in a relatively narrow range of magnetic fields. On the other hand, the results presented in Figs. 2 and 3 confirm that the magnetization reversal of the wedged $\mathrm{Co}^{\mathrm{S}}$ layer takes place mainly by domain wall movement along the direction of the gradient. For the outer voltage contacts 1-2, 2-3, and 8-9, the transition between low and high resistance states is spread over a wider magnetic field interval (Fig. 3). This is in agreement with a previous observation $^{7}$ indicating a more complicated domain structure of wedge shaped Co layers at thicknesses corresponding to the maximum of $H_{\mathrm{C}}$ and for thicknesses in the vicinity of the reorientation transition.

Fig. 4(a) shows the resistance measured using contacts 1 and 9 as a function of the magnetic field. The major loop, recorded up to a magnetic field large enough to saturate both the $\mathrm{Co}^{\mathrm{S}}$ and $\mathrm{Co}^{\mathrm{H}}$ layers, indicates that the magnetic fields sufficient for complete magnetization reversal of the soft layer are distinctly smaller than field necessary to initiate reversal of the hard layer. The other graphs, shown in the same figure, correspond to magnetoresistance measurements. The initial field sequence was the same as that of Fig. 3 but the field was reversed at a preset field $H_{\mathrm{z}}^{\text {rev }}$ and reduced, with the same rate, to zero. The distinct increase of resistance after reversal of the magnetic field is probably caused by thermally activated domain wall movement. ${ }^{19,23}$ Nevertheless the resistance values recorded at remanence $(H=0)$ are directly proportional to the field $H_{\mathrm{z}}^{\text {rev }}$ (Fig. 4(b)).

The individual sensor is capable of registering field along one direction: normal to the film plane. It measures either positive or negative fields, depending on the initial magnetization orientation within magnetic layers (Fig. 4(a)). Thus the proposed magnetoresistive spin valve system can be used as a sensor to determine the magnetic field strength and direction. The magnetoresistance effect in the described system amounts to $\Delta R / R \approx 2 \%$. The relatively small change in resistance is due to the thick buffer, spacer, and cover layers. For real applications, the proposed system can be optimized.
$\Delta R / R$ can be increased by, e.g., using a double spin valve structure $\left(\mathrm{Au} / \mathrm{Co}^{\mathrm{H}} / \mathrm{Au} / \mathrm{Co}^{\mathrm{S}}\right.$-wedge/Au/Co $\left.{ }^{\mathrm{H}} / \mathrm{Au}\right)$ or by adjusting the anisotropy gradient of the wedged layer, i.e., by adjusting the thickness gradient of the $\mathrm{Co}^{\mathrm{S}}$ layer. Nevertheless, the field sensitivity $(0.03 \% / \mathrm{Oe})$ of the sensor system of Fig. 4(b) is comparable to those presented in Ref. 14.

In summary, we have proposed a spin valve system consisting of a $\mathrm{Co}^{\mathrm{H}} / \mathrm{Au} / \mathrm{Co}^{\mathrm{S}}$ trilayer with perpendicular anisotropy of the two Co layers and laterally graded anisotropy of the $\mathrm{Co}^{\mathrm{S}}$ layer to be used as a magnetic field sensor. The functionality of the layer system has been shown, where due to its specific magnetization reversal, the remanent resistance is proportional to the maximum field strength of a magnetic field in a defined direction.

This work was partially supported by the Polish State Committee for Scientific Research, Grant No. N507_287836 and of the Polish Minister of Science and High Education under the Grant IP No. 2011028371.

${ }^{1}$ B. Kirby, J. Davies, K. Liu, S. Watson, G. Zimanyi, R. Shull, P. Kienzle, and J. Borchers, Phys. Rev. B 81, 100405(R) (2010).

${ }^{2}$ J. Davies, P. Morrow, C. Dennis, J. Lau, B. McMorran, A. Cochran, J. Unguris, R. Dumas, P. Greene, and K. Liu, J. Appl. Phys. 109, 07B909 (2011).

${ }^{3}$ R. Dumas, Y. Fang, B. Kirby, C. Zha, V. Bonanni, J. Nogus, and J. Åkerman, Phys. Rev. B 84, 054434 (2011).

${ }^{4}$ Y. Fang, R. Dumas, C. Zha, and J. Åkerman, IEEE Magn. Lett. 2, 5500104 (2011).

${ }^{5}$ R. Dumas, C. Zha, Y. Fang, V. Bonanni, J. Lau, J. Nogués, and J. Åkerman, IEEE Trans. Magn. 47, 1580 (2011).

${ }^{6}$ C. Zha, R. Dumas, Y. Fang, V. Bonanni, J. Nogués, and J. Åkerman, Appl. Phys. Lett. 97, 182504 (2010).

${ }^{7}$ M. Kisielewski, A. Maziewski, Z. Kurant, M. Tekielak, A. Wawro, and L. T. Baczewski, J. Appl. Phys. 93, 7628 (2003).

${ }^{8}$ M. Urbaniak, P. Kuświk, Z. Kurant, M. Tekielak, D. Engel, D. Lengemann, B. Szymański, M. Schmidt, J. Aleksiejew, A. Maziewski, A. Ehresmann, and F. Stobiecki, Phys. Rev. Lett. 105, 067202 (2010).

${ }^{9}$ S. Laribi, V. Cros, M. Muñoz, J. Grollier, A. Hamzić, C. Deranlot, A. Fert, E. Martinez, L. López-Diaz, L. Vila, G. Faini, S. Zoll, and R. Fournel, Appl. Phys. Lett. 90, 232505 (2007).

${ }^{10}$ G. A. Prinz and A. Va, U.S. Patent 5,475,304 (12 December 1995).

${ }^{11}$ I. Tchertkov and S. John, U.S. Patent 5,936,400 (10 August 1999).

${ }^{12}$ R. Coehoorn, K.-M. H. Lensses, and P. J. H. Bloemen, U.S. Patent 6,577,124 B2 (10 June 2003).

${ }^{13}$ A. Taga, L. Nordström, P. James, B. Johansson, and O. Eriksson, Nature (London) 406, 280 (2000)

${ }^{14}$ K. Lee, M.-J. Park, S. Lee, J.-Y. Kim, and B. Cho, J. Appl. Phys. 107, 073905 (2010).

${ }^{15}$ F. Mancoff, J. Dunn, B. Clemens, and R. White, Appl. Phys. Lett. 77, 1879 (2000).

${ }^{16}$ Q. Qin, H. Wei, and X. Han, J. Appl. Phys. 103, 07 E906 (2008).

${ }^{17}$ C. Chappert, K. L. Dang, P. Beauvillain, H. Hurdequint, and D. Renard, Phys. Rev. B 34, 3192 (1986).

${ }^{18}$ M. Speckmann, H. Oepen, and H. Ibach, Phys. Rev. Lett. 75, 2035 (2010).

${ }^{19}$ J. Ferré, J. Jamet, J. Pommier, P. Beauvillain, C. Chappert, R. Mégy, and P. Veillet, J. Magn. Magn. Mater. 174, 77 (1997).

${ }^{20}$ F. Stobiecki, B. Szymański, T. Luciński, J. Dubowik, M. Urbaniak, and K. Röll, J. Magn. Magn. Mater. 282, 32 (2004).

${ }^{21}$ R. Sellmann, H. Fritzsche, H. Maletta, V. Leiner, and R. Siebrecht, Phys. Rev. B 64, 054418 (2001).

${ }^{22}$ M. Urbaniak, F. Stobiecki, B. Szymański, A. Ehresmann, A. Maziewski, and M. Tekielak, J. Appl. Phys. 101, 013905 (2007).

${ }^{23}$ M. Czapkiewicz, T. Stobiecki, and S. van Dijken, Phys. Rev. B 77, 024416 (2008). 